Martz, D. M., Petroff, A. B., Curtin, L.A., Bazzini, D. G. (2009). Gender Differences in Fat Talk Among American Adults: Results from the Psychology of Size Survey. Sex Roles :A Journal of Research, 61(1-2): 34-41. (Jan 2009) Published by Springer Verlag (ISSN: 1573-2762). DOI: 10.1007/s11199-009-9587-7 The original publication is available at www.springerlink.com.

\title{
Gender Differences in Fat Talk Among American Adults: Results from the Psychology of Size Survey
}

\author{
Denise M. Martz, Anna B. Petroff, Lisa Curtin, and Doris G. Bazzini
}

\begin{abstract}
Vignettes were used to assess gender differences in likelihood of hearing others engage in and perceived pressure to join in positive, negative (fat talk), and self-accepting body talk. An agerepresentative sample of 4,014 adult women and men voluntarily responded to an emailed "Health and Wellness" survey from an internet polling company with whom they had preregistered. Women reported more likelihood of hearing fat-talk scenarios and greater pressure to participate in them compared to men. Only a subset of participants reported frequent exposure to and pressure to join in fat talk. Demographic predictors of pressure to engage in fat talk were also examined. This was the first survey to examine body talk among older adults.
\end{abstract}




\section{INTRODUCTION}

Female role models in U.S. media have decreased in body size over the past 50 years, a trend that corresponds negatively with women's body esteem (Grogan et al. 1996; O'Dea 1995; Stice et al. 1994). Conversely, the United States is experiencing record high obesity rates (Flegal et al. 2002; Ogden et al. 2006). A conversational style called "fat talk" gives voice to the typical discrepancy between the average woman's body size and that of the idyllic thin physique. Fat talk was originally defined as the highly ritualized conversation noted among young Caucasian females involving explicit negative self-statements, physical appearance complaints, and weight management tips (Nichter and Vuckovic 1994). Considering the historical context of U.S. culture objectifying women's bodies as valued through their physical appearance (McKinley 2002), we posit that fat talk is a social extension of body objectification. Therefore, we expect that more adult women compared to adult men would report having heard fat talk in their social circles and would report more perceived pressure to join in fat-talk conversations.

This large-scale, age-representative, cross-sectional study of American adults used several vignettes describing others engaging in positive, self-accepting, or negative body talk (fat talk), to assess gender differences and other predictors of hearing such talk and perceived pressure to join in each of these body-focused conversations. This study uniquely examined multiple forms of body talk among adult women and men, extending assessment of body talk beyond the more frequently studied middle school and college years.

Past research indicates that adolescent girls and college students are aware of fat talk. Nichter's (2000) ethnographic study of fat talk in U.S. adolescent females documented an awareness of the social expectation to fat talk when companions were doing so. Similarly, Britton et al. (2006) found that male and female Caucasian college students perceived fat talk as normative by predicting that a hypothetical college woman would say negative things about her own body when exposed to other women doing so.

Nichter (2000) suggested that participation in fat talk may serve multiple functions in female conversation including eliciting social validation, providing an outlet for negative emotions, relieving guilt when eating certain foods, establishing an in-group, and managing one's impression during social conversations. Theoretically, fat talk may be an extension of female conversational tendencies to disclose personal information, to agree with and validate each other, and to communicate personal modesty while acknowledging cultural imperatives that appearance is important for females in a culture that tends to objectify the feminine physique (Carli 1982; Dindia and Allen 1992; Eagly 1987; Janoff-Bulman and Wade 1996; McKinley 2002; Tannen 1990). Nichter (2000) found that some girls thought failing to participate in fat talk would be construed as bragging or egotism based on their perceived pressure to join in. Furthermore, this type of dialog has the potential to create harm. Stice et al. (2003) found that when collegeaged women engaged in a conversation with a thin, attractive confederate who expressed body dissatisfaction, participants felt worse about their own bodies, compared to when exposed to a neutral conversation topic.

Despite growing empirical evidence of fat-talk conversations among young women and the awareness of the norm by young men (Britton et al. 2006), the occurrence of and perceived 
pressure to engage in this phenomenon in the general population is unknown. Britton et al. found that although college women in their study reported they, personally, would not participate in fat talk when other women were doing so, they believed that most women would. Furthermore, Tucker et al. (2007) found evidence of conformity to such pressure in an experimental fat-talk situation involving college-age women.

It is unclear, however, whether American women older than their teens and early twenties experience fat talk situations or feel pressure to engage in these discussions. Concurrently, the question of whether men body talk at all or perceive pressure to participate in body-focused dialogs has been largely ignored. Since previous research suggests that body mass index (BMI) and other demographic variables relate to body image dissatisfaction in U.S. women (Mujahid et al. 2005), we examined whether variables such as BMI or demographic variables predict pressure to engage in fat talk. The current study surveyed an age-representative sample of American adults via the internet to examine gender effects and other predictors of likelihood of hearing different types of body talk (negative [fat talk], self-accepting, positive) during social conversations, and perceived pressure to participate in such discourse. Our main hypotheses were that 1) women would report hearing more fat talk and 2) that women would report more pressure to join in fat-talk conversations compared to men. Within gender comparisons of the different forms of body talk were considered purely exploratory, as there is no previous literature examining other forms of body talk other than fat talk.

\section{METHOD}

\section{Design and Procedure}

The research design was a mixed model with gender as the between-subjects independent variable and type of body talk (negative, self-accepting, and positive talk) as the within-subjects variable. Likelihood of hearing each type of body talk and pressure to join in each type of body talk served as the two dependent variables. Additional exploratory analyses were run to examine if demographic variables predict who experiences the most pressure to join in fat talk conversations.

This study was part of the "Psychology of Size" large-scale cross-sectional descriptive survey sponsored by Slim-Fast ${ }^{\mathrm{TM}}$ and conducted on the MyView Research site of the internet by a polling company named The Segmentation Company, a division of Yankelovich. Participants, who were all legal U.S. citizens and 18+ years of age, were previously enrolled in an online research panel to serve as participants in a variety of polling activities. Sequential email blasts between May 11 and May 18, 2007, titled "Health and Wellness Survey," were sent to this group inviting them to participate according to certain demographic quotas (e.g., age stratification; equal number of males and females). Participants were not told that this survey was sponsored by Slim-Fast ${ }^{\mathrm{TM}}$ or that it was called the Psychology of Size. Consent to participate was inherent in the voluntary completion of the online survey and all participants received a \$1 Pay-Pal ${ }^{\mathrm{TM}}$ reward for their time. Institutional Review Board approval for use of this archival data was received on June 8, 2007. 


\section{Participants}

A total of 4,014 individuals (2,007 women and 2,007 men) participated. Table 1 summarizes demographic information that was assessed on the survey separately for males and females. BMI was calculated based on participants' self-reported weight and height. Participants reported their height and weight, which were later converted into body mass indices (BMl; weight in kilograms/ height in meters squared) and grouped according to Center for Disease Control and Prevention (2007) guidelines. Only $2.4 \%$ of men and $2.8 \%$ of women fell into the underweight category (BMI below 18.5). Thirty percent of men and $31.9 \%$ of women were classified as normal weight (BMI 18.5-24.9), 35.4\% of men and $27.9 \%$ of women met overweight status (BMI 25-29.9), and $32.2 \%$ of men and $37.3 \%$ of women fell into the obese classification (BMI $30+$ ).

Table 1 Percent of men and women falling into each demographic category.

\begin{tabular}{llll}
\hline Characteristic & Men (\%) & Women (\%) & Total (\%) \\
\hline Employment & & & \\
Full-time & 27.4 & 18.3 & 45.7 \\
Part-time & 4.1 & 6.9 & 11.0 \\
Student & 3.5 & 1.8 & 5.3 \\
Retired homemaker & .5 & 9.6 & 10.2 \\
Retired & 10.1 & 9.8 & 20.0 \\
Unemployed & 4.3 & 3.5 & 7.8 \\
Income & & & \\
Less than 34,999 & 12.2 & 16.7 & 28.9 \\
35,000-49,999 & 10.3 & 10.7 & 21.0 \\
50,000-74,999 & 11.3 & 10.9 & 22.2 \\
75,000-99,999 & 6.5 & 5.4 & 11.9 \\
100,000-149,999 & 4.5 & 3.1 & 7.7 \\
Over 150,000 & 2.4 & 1.3 & 3.7 \\
Did not disclose & 2.8 & 1.8 & 4.6 \\
Education & & & \\
S8th grade & .3 & .0 & .4 \\
Some high school & 1.4 & .9 & 2.4 \\
High school graduate & 8.8 & 13.0 & 21.8 \\
Some college & 17.5 & 18.9 & 36.4 \\
College graduate & 14.6 & 12.1 & 26.6 \\
Post-graduate study & 7.4 & 5.0 & 12.4 \\
\hline
\end{tabular}

Mean age $=45$ years $(\mathrm{SD}=16$, Range $18-100) .(N=4,014 ; 2,007$ men and 2,007 women) 


\section{Materials}

The Psychology of Size survey was self-designed by the first author and The Segmentation Company of Yankelovich and contained 130+ items assessing body image, size, and weight concerns for people in the U.S.

The focus of this study was on the fat talk items. Respondents were asked to read and respond to each of the following three scenarios: (1) Fat talk scenario- "Imagine you are in a group of friends/coworkers who were saying negative things about their bodies (For example, "My butt is fat")"; (2) Self-accepting scenario-"Imagine you are in a group of friends/coworkers who were saying self-accepting things about their bodies (For example, "I feel okay about my body")"; (3) Positive scenario-Imagine you are in a group of friends/coworkers who were saying positive things about their bodies (For example, "I really like my body")."

\section{Likelihood Variable}

After reading each scenario, participants were asked to report "how likely would this scenario occur in your life?" using the following Likert-type continuum (1 = never; 2 = sometimes; $3=$ usually; 4 = frequently; $5=$ very frequently).

\section{Pressure Variable}

After reading each scenario, participants were asked to report "how much pressure would you feel to say negative things (changed to "positive things", "self-accepting things" for respective scenarios) about your body in this group?" using the following Likert-type continuum ( 1 = none; $2=$ maybe some; $3=$ some; $4=$ a lot $; 5=$ extreme) .

\section{RESULTS}

\section{Likelihood of Hearing Body Talk Conversations}

Likelihood ratings of occurrence of the three body talk scenarios were analyzed using a 2 (gender) $\times 3$ (body-talk scenario) repeated-measures analysis of variance (RM-ANOVA) with gender as the between-subjects variable and type of body-talk scenario as the within-subjects variable. Supporting our first hypothesis, a significant gender effect occurred with women reporting a greater likelihood of hearing negative and self-accepting talk compared to men, $F(2$, $4011)=298.5, p<.0001, \quad \eta_{P}^{2}=.130$. However, this main effect was qualified by a significant interaction between gender and type of body talk, $F(2,4011)=158.9, p<.0001, \quad \eta_{P}^{2}=.073$. Table 2 illustrates the means, standard deviations, and post hoc comparisons for the body-talk likelihood scenarios between men and women. In order to examine the gender by body-talk- 
scenario interaction, post-hoc, pairwise t-tests were conducted separately for women and men. Women reported more likelihood of negative body talk compared to self-accepting talk, and more likelihood of self-accepting talk compared to positive talk. Men reported equivalent probability of exposure to fat talk and self-accepting talk. They reported less likelihood of positive body talk compared to both negative and self-accepting talk.

Table 2 Means, standard deviations, and comparisons between females and males across type of body talk.

\begin{tabular}{llll}
\hline & $\begin{array}{l}\text { Negative (fat } \\
\text { talk) }\end{array}$ & Self-accept & Positive \\
\hline $\begin{array}{l}\text { Likelihood } \\
\text { Female }\end{array}$ & $2.74(1.25)_{\mathrm{acg}}$ & $2.12(.99)_{\mathrm{ade}}$ & $1.85(.96)_{\mathrm{ath}}$ \\
Male & $2.04(1.05)_{\mathrm{bcg}}$ & $2.03(.98)_{\mathrm{bce}}$ & $1.92(1.01)_{\mathrm{ath}}$ \\
Pressure & & & \\
Female & $2.27(1.19)_{\mathrm{acg}}$ & $2.10(1.06)_{\mathrm{ade}}$ & $1.98(1.08)_{\mathrm{ath}}$ \\
Male & $1.80(1.00)_{\mathrm{bcg}}$ & $1.95(1.04)_{\mathrm{bde}}$ & $1.91(1.07)_{\mathrm{ath}}$ \\
\hline
\end{tabular}

The Likelihood continuum ranged from " $1=$ never" to " $5=$ very frequently. The Pressure continuum ranged from " $1=$ none" to " $5=$ extreme." Significant differences between females and males for Likelihood and Pressure variables separately are indicated by an "a" versus "b" subscript within each column or type of body talk using a one-way ANOVA, $F(1,4012)$ with $p<.05$. There were no gender differences for Positive talk as indicated by shared "a" subscripts. Within-subjects body talk post-hoc comparisons used paired t-tests within gender comparing each type of talk to one another, $t(2007)$ with $p<.05$. Significant differences between negative talk and self-accept talk are indicated by a "c" versus "d" subscript. Significant differences between self-accept and positive talk are indicated by an "e" versus "f" subscript, whereas differences between negative and positive talk are indicated by " $g$ " versus " $h$ " subscripts.

\section{Pressure to Engage in Body Talk Conversations}

An identical RM-ANOVA was conducted for participants' reported pressure to participate in the three types of body talk, yielding the second hypothesized gender main effect with women reporting more pressure than men to engage in negative and self-accepting talk, $F(2$,

$4011)=20.3, p<.0001, \quad \eta_{P}^{2}=.010$. Again this main effect was qualified by a gender by body talk interaction, $F(2,4011)=56.0, p<.0001, \quad \eta_{P}^{2}=.027$. Post-hoc results are presented in Table 2. Women reported more perceived pressure to engage in negative body talk compared 
to self-accepting and positive talk, and reported more pressure to engage in self-accepting talk than positive body talk. Men, on the other hand, reported more pressure for self-accepting talk over negative talk and positive talk. They also reported more pressure for positive talk over negative body talk.

\section{High Likelihood of and Pressure to Body Talk}

Gender differences were reported for likelihood of hearing and pressure to join in different types of body talk conversations. However, the means in Table $\underline{2}$ suggest that the self-reported likelihood of experiencing the varying body-talk situations and perceived pressure to engage in such dialog was relatively low on the Likert continuum (i.e., less than 3 on a five-point scale) and significant differences produced small effect sizes. This suggests that most of these adult participants reported none or just a small likelihood of occurrence and pressure to engage in body-talk conversations. Because the meaning of the Likert continua assessing these constructs was referential rather than criterion based and since the focus of this research centered on who experiences and engages in fat talk, these variables were dichotomized in order to allow us to examine gender differences between participants who reported the most likelihood of hearing and the most perceived pressure to participate in each of the three bodytalk scenarios. Participants who reported " $4=$ frequently" or " $5=$ very frequently" on the likelihood of hearing each body-talk scenario were classified as "high" on likelihood of experiencing the body discussion scenario. Eleven percent of men and 31\% of women reported high exposure to the negative body-talk scenario; $8 \%$ of men and $11 \%$ of women reported a high likelihood of hearing self-accepting body-talk scenario, and $8 \%$ of men and $8 \%$ of women reported high exposure to the positive body-talk scenario.

Similarly, participants were categorized as "high" on perceived pressure to engage in the bodytalk scenario if they responded with " $4=$ a lot" or " $5=$ extreme," yielding the following breakdown: high pressure negative scenario $=7 \%$ of men, $17 \%$ of women; high pressure self-accept scenario $=8 \%$ of men, $10 \%$ of women; high pressure positive scenario $=9 \%$ of men, $10 \%$ of women. High likelihood of and pressure to participate in these different forms of body talk affected only a subset of our sample, and women were more likely than men to report experience with and pressure to participate in negative body talk scenarios.

Consistent with the gender effects from the previous analyses, Fig. 1 demonstrates that of those reporting a high likelihood of experiencing a positive talk scenario, nearly half were men (50.8\%) and nearly half were women (49.2\%). Of participants endorsing a high likelihood of occurrence of self-accepting body talk, $43.4 \%$ were men and $56.6 \%$ were women. Finally, a pronounced difference was noted among participants endorsing a high likelihood of hearing a negative body talk scenario with $73.6 \%$ being women and $26.4 \%$ being men. 


\section{Gender Differences 29}

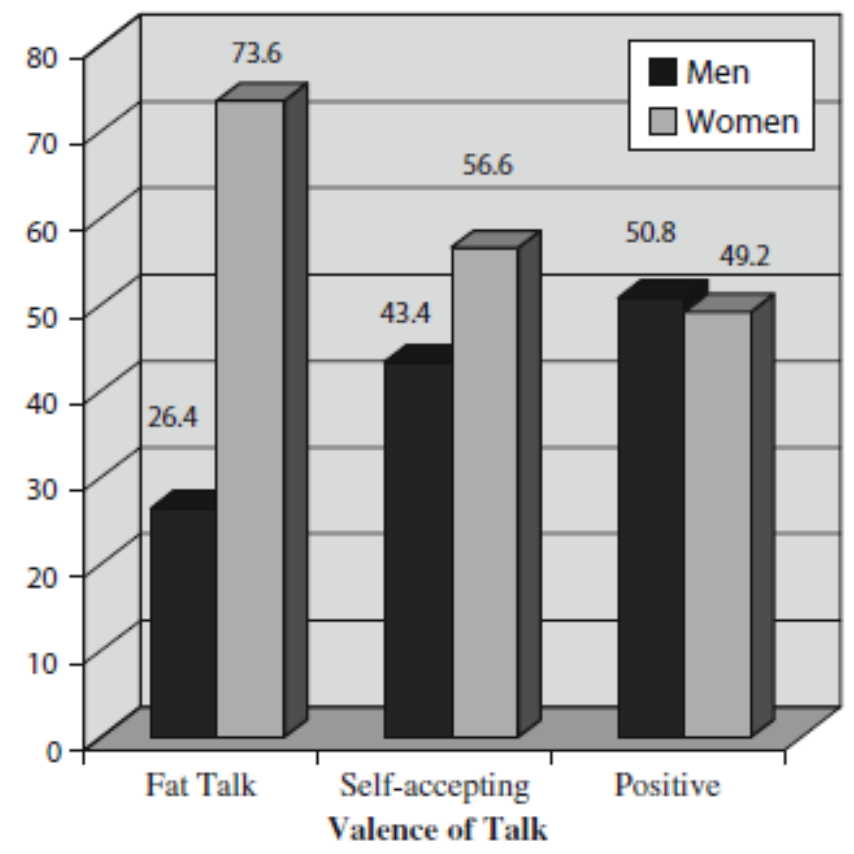

Figure 1. Percentage of participants reporting high likelihood hearing each type of body talk by gender.

Figure 2 shows those reporting high pressure to participate in each form of body-talk by gender. A modest difference emerged for the percentage of males (46.8\%) compared to females (52.2\%) falling into the high pressure to conform to positive body talk. Similarly, slightly more of those reporting high pressure to conform to self-accepting body talk were women (55\%) than were men (45\%). Consistent with our findings for high reported likelihood of the negative bodytalk scenario, $71.9 \%$ of participants reporting high pressure to take part in negative body-talk conversations were women and $28.1 \%$ were men. 


\section{Gender Differences 30}

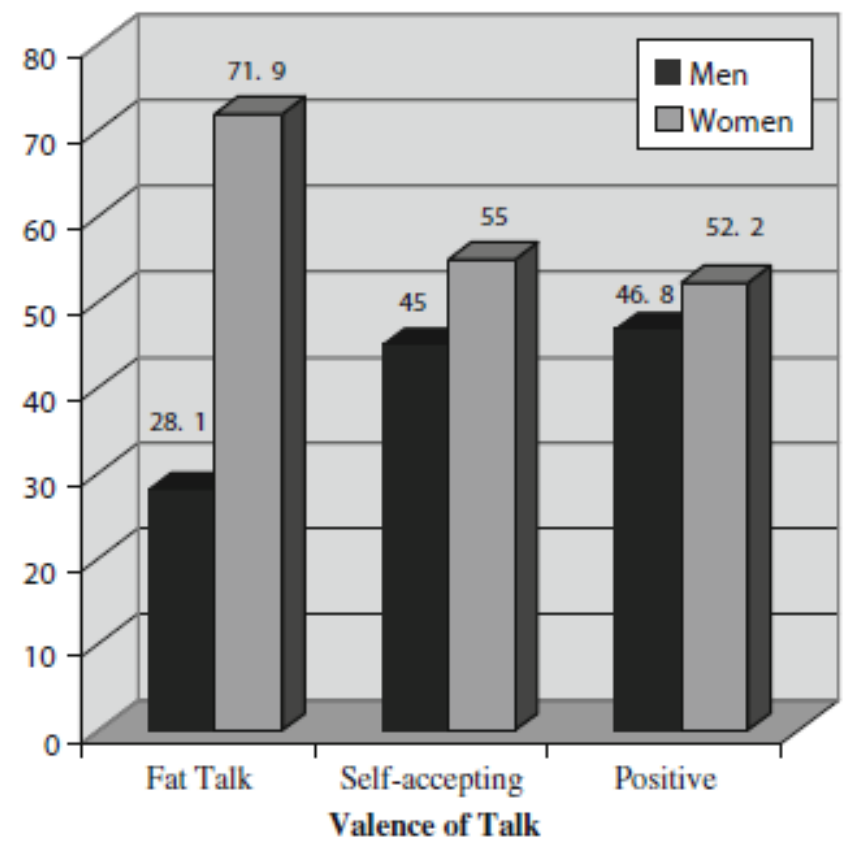

Figure 2. Percentage of participants reporting high pressure to join in each type of body talk by gender.

\section{Demographic Factors Related to Negative Body Talk in Men}

Reported likelihood of negative body talk in one's social circles also positively related to perceived pressure to participate in this type of conversation for men, $r(2,005)=.509, p<.001$. Age was again negatively related to perceived pressure to fat talk, $r(2,005)=-.189, p<.001$, and education level, $r(2,005)=-.035, p=.121$, and income level, $r(2,005)=-.003, p=.905$, did not correlate with pressure to engage in negative body talk among men. Male participants were divided into four groups of increasing BMI (i.e., underweight, normal, overweight and obese) and a one-way ANOVA across levels of BMI showed that it did not relate to pressure to fat talk, $F(3,1,982)=2.29, p=.007$, or men's reported likelihood of exposure to fat talk, $F(3$, $1,982)=1.16, p=.324$. The six employment status categories served as an independent variable in an ANOVA with perceived pressure to fat talk as the dependent variable and similarly yielded a significant equation, $F(5,2,001)=5.55, p<.0001$. Post hoc analyses showed that those men who were retired reported less pressure to fat talk compared to those classified as full time employment, part time employment, student, and not employed.

\section{DISCUSSION}

Literature on the social psychological aspects of body image, or how people respond to and impression manage body-related social conversations, is in the seminal stages. Previous 
research on fat talk has focused almost exclusively on female targets and on middle school and college students, and it has not examined fat talk relative to alternative forms of body talk. This large-scale, age-representative, cross-sectional study of American adults used several hypothetical vignettes depicting others engaging in positive, self-accepting, or negative body talk (fat talk), to assess the relationship between gender and the perceived likelihood of occurrence and pressure to participate in these conversations. Consistent with our hypothesis, women reported a greater likelihood of exposure to fat talk in their social circles and more perceived pressure to join in fat talk compared to men. Of those reporting the highest likelihood of hearing negative body talk, $73.6 \%$ were women, and of those reporting high pressure to engage in fat talk, $71.9 \%$ were women. However, only a subset of female participants reported frequent likelihood of (31\%) and high-perceived pressure to join in fat talk (17\%), a finding that is contrary to some interpretations of Britton et al. (2006) by the popular press that fat talk is "mandatory" in American culture (Goudarzi 2007). Thus fat talk remains more of a feminine cultural phenomenon, yet not all women are affected by it.

Nichter's (2000) original ethnographic research described fat talk as a method for teen females to fit in, and to affiliate and bond with one another. Britton et al. (2006) further found that American college students expected young adult women to derogate their bodies in a group of women engaging in fat talk. Our gender comparisons suggest that fat talk, is indeed, more of a female cultural phenomenon even beyond the teen and college age years. Pressure to fat talk among some women may be a function of feminine communication styles whereby females disclose personal information, affirm one other, and communicate modesty and humbleness (Carli 1982; Dindia and Allen 1992; Eagly 1987; Janoff-Bulman and Wade 1996; Tannen 1990) or part of a larger cultural norm promoting body objectification for American women more so than for men (McKinley 2002). Engaging in fat talk may be a way for some women to acknowledge allegiance to cultural norms that appearance is important and as a social admission that one's body fails to meet a stringently thin ideal. Consistent with objectification theory, our findings showed women fat talk more than men, but our gender comparisons also unexpectedly found women reported more pressure to engage in self-accepting body talk than men. Expressing self-acceptance of one's body image continues a norm of body objectification, but is certainly more positive than public degradation of one's physique. This latter finding is consistent with recent research demonstrating that there could be a competing norm for women to express self-acceptance of body image, rather than always fat talk when social conversations focus on body image (Tompkins et al. Unpublished manuscript).

Additionally, we found that gender effects depended on type of body talk. Given the propensity for women to experience greater body dissatisfaction relative to men (Pruzinsky and Cash 2002), our within gender results indicate that women report greater likelihood of hearing fat talk and more pressure to engage in fat talk over other forms of body talk. This finding is consistent with cultural gender differences in body image. Men showed somewhat of an opposite effect in that they reported more pressure to engage in positive or self-accepting body talk over fat talk.

Although not demonstrated empirically in this study, we could hypothesize that actual body image perceptions correspond with social projections of that body image. If this is true, we would expect to hear more fat talk from individuals with poor body image and more self- 
accepting and positive body talk from those with positive body image. The original body image research of Fallon and Rozin (1985), which had college students rate their current and ideal bodies using figure drawings, found that men tended to perceive their current and ideal as similar, whereas women rated themselves as larger than their smaller ideal. Decades of literature since this original research has affirmed continued body image dissatisfaction for Caucasian American women compared to men (Striegel-Moore and Franko 2002). Thus, the gender differences that we found for probability of hearing and pressure to join in fat talk may be a function of gender differences in personal body image perceptions, and perhaps, body size for women.

We found that perceived pressure to participate in negative-body talk clearly related to likelihood of hearing it for both women and men. Although not tested directly in this survey, it is likely that predicting the "likelihood" of a body talk "scenario in your life" relates to past exposure to these social conversations. Moreover, perceived pressure to join in any type of conversation is likely a function of the base-rate of exposure to that particular form of discourse. However, when Britton et al. (2006) asked female college students how a female target would respond in a group of three other women engaging in fat talk, a strong majority reported that she would join in. Yet, these same respondents did not predict that they would follow suit in that situation. Although reported likelihood of exposure to fat talk in social conversations clearly relates to pressure to engage in it, they are not synonymous.

\section{Other Predictors of Pressure to Engage in Fat Talk}

Variables other than gender and exposure to fat talk, such as age, body size, and employment status also related to perceived pressure to engage in fat talk. Considering that fat talk was originally documented in middle school-aged and college-aged samples of females, our data similarly suggests that age was slightly negatively related to pressure to engage in negative body talk for both men and women. We refer to literature related to body image changes throughout the female lifespan to speculate about this result. McKinley (2006) conducted a 10year follow-up study of female undergraduates and their mothers to examine changes in body image longitudinally and found younger women's body shame and surveillance decreased over this time, making them similar to the middle-aged women. This psychological change occurred despite weight gain in that cohort. She attributed this improvement of body image to typical life transitions that follow college, including the formation of committed relationships and establishment of careers and families. Thus, some research supports the theory that American women experience improvements in body image as they age. If pressure to fat talk is associated with personal feelings about one's body, perhaps this is the reason that increased age related to slightly less pressure to engage in fat talk. Or since pressure to fat talk was related to reported likelihood of hearing it, it is possible that older women think they will hear it less and therefore, their perceived pressure to conform is diminished. It is unclear why age was slightly negatively related to pressure to engage in fat talk for our male participants, and we

were unable to find longitudinal body image research on American men. Our finding that male and female participants who classified themselves as retired reported less likelihood and 
pressure to join in fat talk is likely related to age in that retirees tend to be older. Moreover, retirees may find themselves in fewer appearance-related discussions about body image.

Future research might examine how cohort effects or maturation and employment status affect adults' body talk and gender interactions in Western culture.

Body mass index also predicted reported pressure to engage in fat talk with our obese women, and to some extent our overweight women, reporting greater pressure than normal weight or underweight individuals. Yet our larger women did not report more occurrences of negative body talk in their social situations. There was no relationship between weight status and likelihood of hearing or pressure to engage in fat talk for our male participants, however. In U.S. culture, individuals who are overweight or obese face pernicious stigmatization (Myers and Rosen 1999). Unlike racial prejudice, this stigma is often overtly expressed because body weight is considered controllable (Crandall 1994). Perhaps when larger women are exposed to a social situation involving fat talk, they feel pressure to acknowledge their size and the visible contrast to the cultural ideal. Thus, it is possible that larger women would not deliberately initiate such conversations, yet still need to impression manage when others are engaging in this dialog. Future studies may want to examine more closely how body size of speaker and body sizes of audience impact varying forms of body talk in social conversations.

\section{Limitations}

Although the present survey uniquely employed a large number of male and female American adults as participants to examine fat talk, the cross-sectional design does not allow for examination of causal relationships or relationships between variables across time. Moreover, assessment of likelihood of hearing body talk and perceived pressure to join body-talk conversations relied on self-reported responses to brief fictional vignettes. Future research should examine whether self-reports of different forms of body talk correspond with actual behaviors in social situations, and if and when, there is a difference between self-reports and observed behavior. Additionally, participants were asked to imagine scenarios of varying body talk among friends or coworkers. We did not specify the gender(s) of said friend/coworkers. This aspect of the study may be a limitation because there could be a gender difference in the imagined audience for any type of body talk. Perhaps the seemingly feminine nature of body talk led our female participants to envision all female configurations of friends and coworkers, whereas some of the men may not have been imagining all male configurations, but rather mixed company, when envisioning their friends and coworkers.

Additionally, we chose the phrase "my butt is fat" as the example for our negative body talk scenario and retrospectively, acknowledge this may be an inherently feminine example of fat talk. Considering that overweight men tend to carry adiposity surrounding the torso, it is questionable if men, overweight or not, would identify with our example. Moreover, targeting one body part in this example may have failed to capture the more general perception of emotional and physical dissatisfaction of females when they voice "I am so fat" (Nichter 2000). Future research should manipulate gender of audience to determine how gender variations relate to and impact body-talk conversations. 
Finally, we assessed participants' likelihood of hearing different forms of body talk in their life and perceived pressure to join in these conversations as our key dependent variables. It is unclear if participants' estimates of likelihood of occurrence of any type of conversation is a function of previous experience with and exposure to those conversations. Moreover, perceived pressure to engage in a behavior may be very different than actual behavior once an individual is placed within a real social environment. As of this date, most investigations of fat talk have asked participants to place themselves into hypothetical conversations and to report how they think they would behave and how they think others would behave (Britton et al. 2006), or have asked participants to publicly rate their perceived body image in front of a confederate (Tucker et al. 2007). Hence, research on fat talk is in its infancy as are measures attempting to capture such constructs. We recommend that future research examine the behavioral validity of any self-reported measure of body talk.

\section{Implications}

Research on fat talk, especially relative to alternative forms of body talk, is in its formative years. Nichter and Vuckovic (1994) coined the term and documented it in middle-school Caucasian girls (Nichter 2000). Britton et al. (2006) showed that both male and female college students, presented with a vignette of females engaging in fat talk, thought that the average woman would also derogate her own body. However, we found that only $31 \%$ of our age-representative sample of adult American women and $11 \%$ of men reported a high likelihood of occurrence of fat talk in their social circles. It could be that social variables unique to middle school (e.g., locker room changing) and the college atmosphere facilitate the occurrence of fat talk conversations. Conversely, the typical maturation process beyond young adulthood may buffer body dissatisfaction, therefore lessening its expression through fat talk, in social situations (McKinley 2002). We propose that social psychological examination of how culture relates to body image, particularly individuals' reflection and impression management of that body image, and how that may promote and influence bidirectionally their appraisal of personal body image, is deserving of future research attention.

\section{REFERENCES}

Britton, L. E., Martz, D. M., Bazzini, D. G., Curtin, L. A., \& LeaShomb, A. (2006). Fat talk and self-presentation of body image: is there a social norm for women to self-degrade? Body Image: An International Journal of Research, 3, 247-354.

Carli, L. L. (1982). Are women more social and men more task oriented? A meta-analytic review of sex differences in group interaction, reward allocation, coalition formation, and cooperation in the Prisoner's Dilemma game. Unpublished manuscript, University of Massachusetts, Amherst.

Centers for Disease Control and Prevention. (2007). About BMI for Adults. Retrieved May 4, 2008, from http://www.cdc.gov/nccdphp/dnpa/bmi/adult_BMI/about_adult_BMI.htm\#Interpreted. 
Crandall, C. S. (1994). Prejudice against fat people: Ideology and self-interest. Journal of Personality and Social Psychology, 66, 882-894.

Dindia, K., \& Allen, M. (1992). Sex differences in self-disclosure: a meta-analysis. Psychological Bulletin, 112, 106-124.

Eagly, A. H. (1987). Sex differences in social behavior: A social-role interpretation. Hillsdale, NJ: Lawrence Erlbaum.

Fallon, A. E., \& Rozin, P. (1985). Sex differences in perceptions of desirable body shape. Journal of Abnormal Psychology, 94, 102-105.

Flegal, K. M., Carroll, M. D., Ogden, C. L., \& Johnson, C. L. (2002). Prevalence and trends in obesity among U.S. adults, 1999-2000. Journal of the American Medical Association, 288, 1723-1727.

Grogan, S., Williams, Z., \& Conner, M. (1996). The effects of viewing same-gender photographic models on body-esteem. Psychology of Women Quarterly, 20, 569-575.

Goudarzi, S. (2007, March 14). Female Fat Talk Socially Mandatory, Study Finds. Retrieved May 4, 2008, from http://www.msnbc.msn.com/id/17600911/.

Janoff-Bulman, R., \& Wade, M. B. (1996). The dilemma of self-advocacy for women: Another case of blaming the victim? Journal of Social and Clinical Psychology, 15, 143-152.

Mujahid, M. J., Diez-Roux, A. V., Borrell, L. N., \& Nieto, J. (2005). Cross-sectional and longitudinal associations of BMI with socioeconomic characteristics. Obesity Research, 13, 1412-1421.

McKinley, N. (2002). Feminist perspectives and objectified body consciousness. In T. F. Cash, \& T. Pruzinsky (Eds.), Body image: A handbook of theory, research, and clinical practice (pp. 55-62). New York: Guilford.

McKinley, N. M. (2006). The developmental and cultural contexts of objectified body consciousness: A longitudinal analysis of two cohorts of women. Developmental Psychology, 42(4), 679-687.

Myers, A., \& Rosen, J. C. (1999). Obesity stimatization and coping: Relation to mental health symptoms, body image, and self-esteem. International Journal of Obesity, 23, 221-230.

Nichter, M. (2000). Fat talk. What girls and their parents say about dieting. Cambridge, MA: Harvard University Press.

Nichter, M., \& Vuckovic, N. (1994). Fat talk. In N. Sault (Ed.), Many mirrors: Body image and social relations (pp. 109-131). New Brunswick, NJ: Rutgers University Press. 
O'Dea, J. A. (1995). Body image and nutritional status among adolescents and adults-A review of the literature. Australian Journal of Nutrition and Dietetics, 52, 56-68.

Ogden, C. L., Carroll, M. D., Curtin, L. R., McDowel, M. A., Tabak, C. J., \& Flegal, K. M. (2006). Prevalence of overweight and obesity in the United States, 1999-2004. Journal of the American Medical Association, 295, 1549-1555.

Pruzinsky, T., \& Cash, T. F. (2002). Understanding body images: Historical and contemporary perspectives. In T. F. Cash, \& T. Pruzinsky (Eds.), Body image: A handbook of theory, research, and clinical practice (pp. 3-12). New York: Guilford.

Stice, E., Schupak-Neuberg, E., Shaw, H. E., \& Stein, R. I. (1994). Relation of media exposure to eating disorder symptomatology: An examination of mediating mechanisms. Journal of Abnormal Psychology, 103, 836-840.

Stice, E., Maxfield, J., \& Wells, T. (2003). Adverse effects of social pressure to be thin on young women: An experimental investigation of the effects of "fat talk.". The International Journal of Eating Disorders, 34, 108-117.

Striegel-Moore, R. H., \& Franko, D. L. (2002). Body image issues among girls and women. In T. F. Cash, \& T. Pruzinsky (Eds.), Body image: A handbook of theory, research, and clinical practice (pp. 183-191). New York: Guilford.

Tannen, D. (1990). You Just Don't Understand: Women and Men in Conversation. NewYork: Ballentine Books.

Tompkins, K. B., Martz, D. M., Rocheleau, C., \& Bazzini, D. (Unpublished manuscript). Social likeability, conformity, and body talk: Does fat talk have a normative rival in female body image conversations?

Tucker, K. L., Martz, D. M., Curtin, L. A., \& Bazzini, D. G. (2007). Examining "fat talk" experimentally in a female dyad: How are women influenced by another woman's body presentation style? Body Image: An International Journal of Research, 4, 157-164. 